\title{
Localized magnetic fields enhance the field sensitivity of the gyrotropic resonance frequency of a magnetic vortex
}

\author{
Jasper P. Fried and Peter J. Metaxas* \\ School of Physics, M013, University of Western Australia, 35 Stirling Highway, Crawley, Western Australia 6009, Australia
}

(Received 13 November 2015; revised manuscript received 3 January 2016; published 22 February 2016)

\begin{abstract}
We have carried out micromagnetic simulations of the gyrotropic resonance mode of a magnetic vortex in the presence of spatially localized and spatially uniform out-of-plane magnetic fields. We show that the field-induced change in the gyrotropic mode frequency is significantly larger when the field is centrally localized over lengths which are comparable to or a few times larger than the vortex core radius. When aligned with the core magnetization, such fields generate an additional confinement of the core. This confinement increases the vortex stiffness in the small-displacement limit, leading to a resonance shift which is greater than that expected for a uniform out-of-plane field of the same amplitude. Fields generated by uniformly magnetized spherical particles having a fixed separation from the disk are found to generate analogous effects except that there is a maximum in the shift at intermediate particle sizes where field localization and stray field magnitude combine optimally to generate a maximum confinement.
\end{abstract}

DOI: 10.1103/PhysRevB.93.064422

\section{INTRODUCTION}

A magnetic vortex is a curled magnetization configuration with an out-of-plane magnetized nanoscale-sized core [1-3]. Vortices arise spontaneously in (sub)micron-scale [4] magnetic elements such as disks (as well as square [5] or triangular [6] plates) and are of relevance for a range of applications ranging from radio frequency signal generation [7,8] and detection [9] to cancer treatment [10], data storage [11], and magnonics [12-14]. Many applications exploit the lowest frequency magnetic excitation of a vortex, the gyrotropic mode [15-18], which corresponds to an orbit-like motion of the vortex core about the disk's center.

An important characteristic of the gyrotropic mode is that its frequency, $f_{G}$, can be tuned by applying static out-of-plane $[7,8,19,20]$ or in-plane $[6,21]$ magnetic fields. Uniform out-ofplane magnetic fields modify both the element's magnetization configuration and the magnetostatic confinement of the core and, when sufficiently far below the saturation field of the disk, induce a change in $f_{G}$ which is a linear function of the field strength $[7,8,19]$. The combination of this field linearity with the ability to electrically probe vortex dynamics (by fabricating a vortex-based spin torque nano-oscillator [7,8,22], STNO) has application not only for field-tunable electronic oscillators $[7,8,20,23]$ but potentially also for intrinsically frequencybased submicron magnetic field sensors [24,25]. The latter typically exploit the field dependence of the output frequency of a STNO (e.g., see [24-26] for non-vortex-based devices) for frequency-based [24,27-30] field sensing. One potential application of such a sensor is in the development of frequencybased nanoscale devices to detect (biofunctionalized) magnetic nanoparticles [30] (MNPs) for in vitro biosensing and pointof-care medical diagnostics [31].

In this work we show that central, localized out-of-plane fields (such as those generated by MNPs) produce shifts in the gyrotropic frequency greater than those induced by uniform

\footnotetext{
*peter.metaxas@uwa.edu.au
}

out-of-plane fields having the same amplitude. It is shown that this is due to an increase in the vortex stiffness as a result of the out-of-plane magnetization of the core preferentially aligning with the strongest part of the localized field. For the particular case of MNPs whose lower surfaces are separated from the disk by a fixed distance, we demonstrate that the frequency shift is characterized by a clear maximum at intermediate particle sizes, a result of an optimized combination of the amplitude and localization of the stray field created by the MNP. Finally, we note that short-range structural disorder (as well as domaingenerated stray fields [32] or disk thickness steps [33]) can also pin vortices [34] (with a potential for quantum depinning $[35,36])$ and that these "defects" have also been shown to be capable of modifying the gyrotropic mode frequency [8,33,37-41].

\section{MICROMAGNETIC SIMULATION}

Our results were obtained using finite-difference micromagnetic simulations of the gyrotropic mode using MuMax3 [42]. We will focus on simulation results for NiFe-like disks with radius $R=192 \mathrm{~nm}$, thickness $L=30 \mathrm{~nm}$, saturation magnetization $M_{\mathrm{S}}=800 \mathrm{kA} / \mathrm{m}$, exchange stiffness $A_{\text {ex }}=13 \mathrm{pJ} / \mathrm{m}$, magnetic damping $\alpha=0.008$, no intrinsic anisotropy, and a cell size of $2 \times 2 \times 3.75 \mathrm{~nm}^{3}$. To begin with, the system is initialized with a vortex-like magnetic configuration and allowed to relax using MuMax3's internal relaxation routine which time-evolves the magnetization (without precession) using energy- and then torque-minimization as stopping criteria [42]. A transverse magnetic field sinc pulse is then applied with an amplitude of $2 \mathrm{mT}$, a time offset of $300 \mathrm{ps}$, and a cutoff frequency of $30 \mathrm{GHz}$. This induces a displacement of the vortex core (as well as some higher frequency excitations [21,43-47]) which is followed by a damped gyrotropic motion of the core around the disk's center. Fourier analysis of the $x$ component of the spatially averaged magnetization is used to extract $f_{G}$. Good agreement between MuMax3, OOMMF [48], and FinMag (derived from Nmag [49]) was confirmed for a number of test cases [50]. 


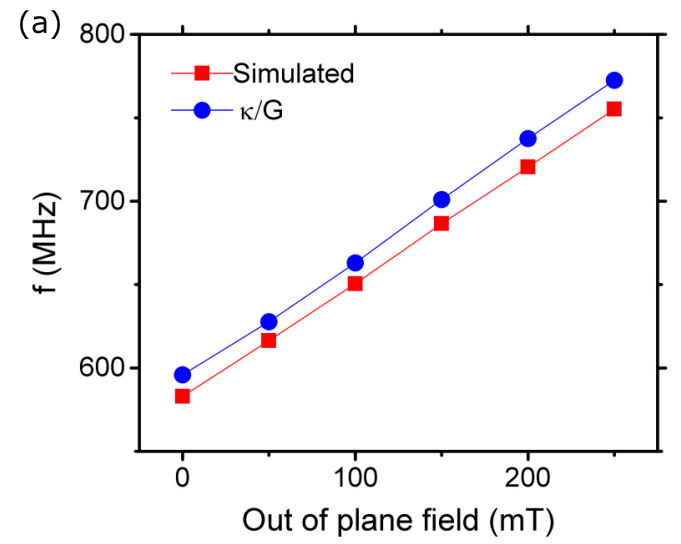

(b)
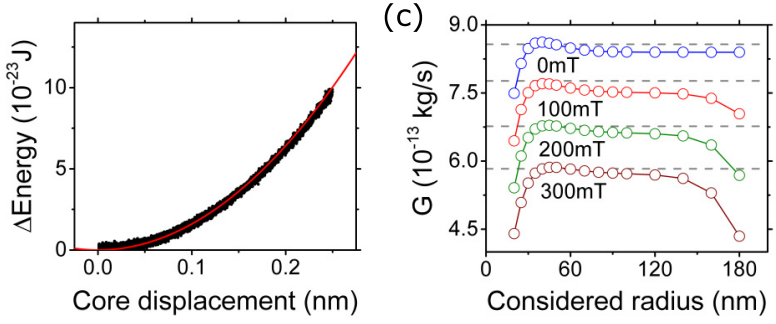

(d)

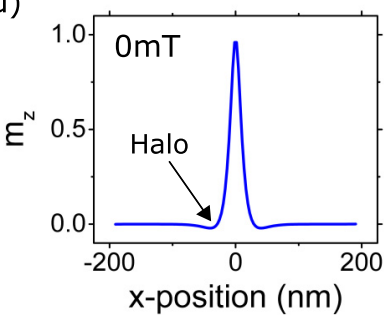

(e)

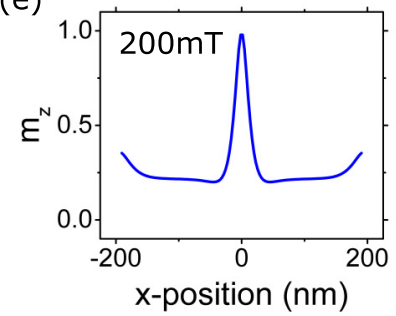

FIG. 1. (a) Simulated and calculated gyrotropic mode frequencies, $f_{G}$ [the latter calculated using Eq. (1)], for spatially uniform out-of-plane fields. (b) Plot of the change in energy (relative to the energy at zero core displacement) as a function of core displacement as measured during field-pulse-induced gyrotropic motion in zero out-of-plane field. The first $25 \mathrm{~ns}$ of motion is disregarded due to the high-frequency spin waves produced in this period. A quadratic fit to the energy is shown by the red curve. (c) Gyroconstant calculated considering different radii from the disk center for four different uniform out-of-plane fields. The gray dashed lines represent the value of the gyroconstant calculated using [19] $G(H)=G(0)(1-\cos \theta)$ where $\cos \theta$ is taken at the minimum of the magnetostatic halo surrounding the vortex core. (d) Out-of-plane magnetization for a centered (nondisplaced) vortex along a slice through the disk's center in zero out-of-plane field and (e) under a spatially uniform $200 \mathrm{mT}$ out-of-plane field.

\section{UNIFORM OUT-OF-PLANE MAGNETIC FIELDS}

We will first consider the case of a vortex in a spatially uniform out-of-plane field [19], $H_{\text {uni. }}$. Figure 1(a) shows the simulated gyrotropic frequency under various amplitude outof-plane fields aligned with the vortex core. As previously observed, the frequency varies linearly with $H_{\text {uni }}$. We were able to quantitatively reproduce the simulated frequencies to within 2\% [blue circles in Fig. 1(a)] using [15]

$$
2 \pi f_{G}=\frac{\kappa}{G},
$$

where $\kappa$ is the vortex stiffness coefficient and $G$ is the gyroconstant. The remainder of this section is focused on the extraction of $\kappa$ and $G$ to obtain the calculated $f_{G}=\kappa /(2 \pi G)$ values shown in Fig. 1(a).

$\kappa$, the stiffness coefficient, describes the harmonic scaling of the vortex energy, $W$, with lateral core displacement, $X$, measured radially from the disk's center: $W(X)=W(0)+$ $\frac{1}{2} \kappa X^{2}+O\left(X^{4}\right)$. For uniform or null out-of-plane fields, this confinement is dominated by dipolar effects due to magnetic charges created during the (dynamic) core displacement [18]. Dynamic exchange fields are also present. For each simulation, we extracted $\kappa$ from a parabolic fit to the total energy of the system plotted against the dynamic displacement of the core as measured during field-pulse-induced gyrotropic motion [e.g., Fig. 1(b)]. This approach analyzes the energy of the dynamic system during core motion, producing a more accurate prediction [21] of the gyrotropic frequency than a static method in which the total energy is calculated for field-displaced, static cores at equilibrium. This said, the static method will be instructive in visualizing the influence of localized fields on the vortex stiffness.

The gyroconstant, $G$, determines the magnitude of the gyrovector, $\mathbf{G}=G \hat{\mathbf{e}}_{Z}$, in the Thiele equation describing vortex dynamics $[15,51-53]$. The gyroconstant can be calculated from the vortex spin structure using

$$
G=\frac{M_{s} L}{\gamma} \iint_{A} \mathbf{m} \cdot\left(\frac{d \mathbf{m}}{d x} \times \frac{d \mathbf{m}}{d y}\right) d x d y,
$$

where $\gamma$ is the gyromagnetic ratio and $\mathbf{m}$ is the unit-length magnetization vector. Given that $\mathbf{G}$ acts along the $z$ axis this equation can be shown to be equal to the often quoted $[15,52] \mathbf{G}=M_{S} L / \gamma \iint \sin (\theta)(\nabla \theta \times \nabla \phi) d x d y$, where $\theta$ and $\phi$ are the polar and azimuthal angles of the magnetization respectively. Theoretically, for a zero out-of-plane field Eq. (2) yields $G=2 \pi q p L M_{s} / \gamma$ where $q=1$ is the vorticity and $p= \pm 1$ is the core polarity aligned along $\pm \hat{\mathbf{e}}_{Z}$.

We numerically calculated the gyroconstant associated with the static, nondisplaced core for the studied $H_{\text {uni }}$ values using Eq. (2) by integrating a thickness-averaged $\mathbf{m}$ [54] over the entire disk area. However, inserting this calculated value of $G$ into Eq. (1) led to a value for $f_{G}$ which was significantly higher than the simulated value. For example, for a uniform out-of-plane field of $200 \mathrm{mT}$ the gyroconstant calculated using this method is $\approx 36 \%$ smaller than what is expected taking the simulated frequency and extracted $\kappa$ and solving for $G$ (i.e., $5.17 \times 10^{-13} \mathrm{~kg} \mathrm{~s}^{-1}$ compared to $7.03 \times 10^{-13} \mathrm{~kg}$ $\left.\mathrm{s}^{-1}=\kappa / 2 \pi f_{G}\right)$. To attempt to understand this discrepancy, we calculated $G$ by considering only the spin structure within a given radius of the disk center for four different $H_{\text {uni }}$ values. The resultant data are shown in Fig. 1(c). $G$ reaches a clear maximum when integrating over a radius close to the edge of the vortex core $(\approx 40 \mathrm{~nm})$. Notably, at this point, the value of $G$ closely corresponds to the gyroconstant expected from the simulated frequency and extracted stiffness coefficient. This peak is present for all out-of-plane field amplitudes and is due to the "magnetostatic halo" [see Fig. 1(d)] surrounding the vortex core as a result of its demagnetization field [55]. The drop-off in $G$ at large radii for $H_{\text {uni }}>0$ is due to the out-of-plane canting of spins near the disk edge [e.g., Fig. 1(e)]. Encouragingly, the maximum value of $G$ calculated using 
this method corresponds closely to the value predicted by the equation $G(H)=G(0)(1-\cos \theta)$ given by de Loubens et al. [19] for uniform out-of-plane fields. We show the values of $G$ calculated using this expression by the gray dashed lines in Fig. 1(c). However, here $\cos \theta$ has been extracted at the center of the magnetostatic halo rather than at the disk's lateral boundary (as specified in Ref. [19]). This result suggests that although there is a divergence of the magnetization far away from the vortex core, it is the local spin structure around the core which is relevant for the small-amplitude oscillations considered here $(<1 \mathrm{~nm})$.

In Fig. 1(c) we see that $G$ reduces considerably when $H_{\text {uni }}$ is increased and it is this reduction in $G$ which is primarily responsible for the linear increase in $f_{G}$ with increasing $H_{\text {uni }}$.

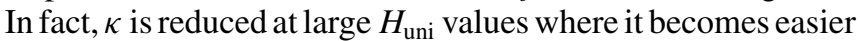
to shift the vortex core laterally. Below, localized out-of-plane fields will also be shown to increase $f_{G}$; however that increase will be demonstrated to be primarily due to an increase in $\kappa$ induced by the localized field.

\section{EFFECT OF SPATIALLY LOCALIZED FIELDS}

\section{A. Gaussian fields}

To study the effect of spatially localized out-of-plane fields on the gyrotropic frequency, we first consider centralized fields with a two-dimensional (2D) Gaussian-like profile:

$$
H_{\mathrm{loc}}=A_{0} \exp \left[-\left(\frac{r}{w_{\mathrm{loc}}}\right)^{2}\right] \text {. }
$$

Here $w_{\text {loc }}$ is a width parameter approximately equal to 1.2 times the half-width at half-maximum (HWHM) of the field profile and $r$ is the lateral distance from the center of the disk. $\mu_{0} A_{0}$ is fixed at $+200 \mathrm{mT}$ making $H_{\text {loc }}$ aligned with the vortex polarity $(p=+1)$. As shown in Fig. 2(a) localized Gaussian fields significantly increase $f_{G}$ as compared to the action of a spatially uniform $200 \mathrm{mT}$ out-of-plane field [gray dashed line in Fig. 2(a)]. Furthermore, this frequency enhancement becomes larger as the Gaussian field becomes more localized.

To begin to understand the above frequency behavior (and confirm its link to a $H_{\text {loc }}$-induced confinement), we first look at how the vortex core moves laterally in response to static, uniform in-plane fields, $H_{\mathrm{IP}}$, in the presence of a centrally localized field, $H_{\text {loc }}$. By doing this we can explicitly probe the (static) confinement of the core across the disk. In the complete absence of an out-of-plane field, the core position varies linearly with $H_{\mathrm{IP}}$ at low fields (low displacements) [Fig. 2(b)]. For higher displacements the response to field is slightly weaker, consistent with an increased (anharmonic [56]) confinement when the core moves closer to the disk's edge under the action of $H_{\mathrm{IP}}$. If we add a localized field however, the $H_{\mathrm{IP}}$-induced core displacement is clearly lower, but only if the displacement is comparable or smaller than the HWHM of the localized field. Indeed, at larger displacements, the response to $H_{\mathrm{IP}}$ is similar for localized out-of-plane fields and zero out-of-plane field. This result explicitly confirms the $H_{\text {loc }}$-induced confinement (or stiffening) of the vortex core which arises because keeping the core within the central region minimizes the Zeeman energy associated with the interaction between $H_{\text {loc }}$ and the vortex core magnetization.

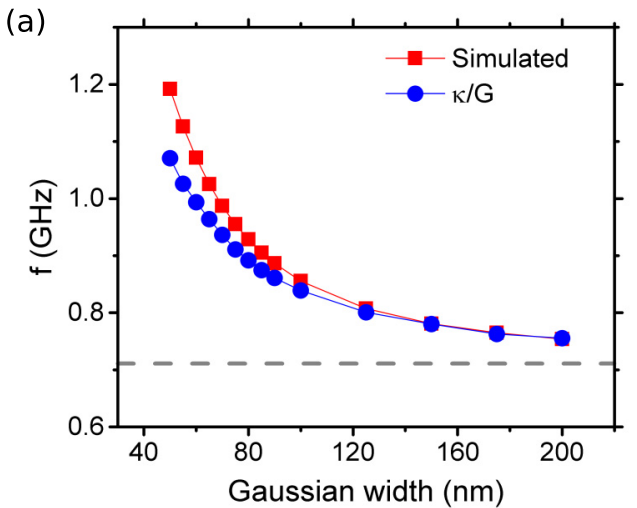

(b)

(d)

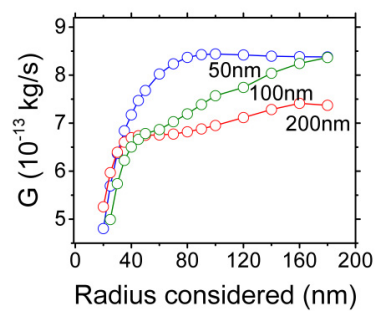

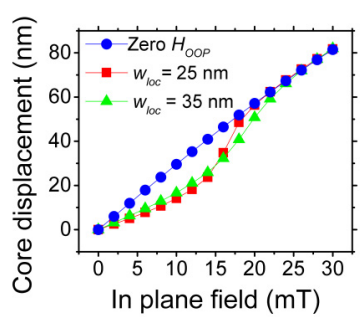

(c)

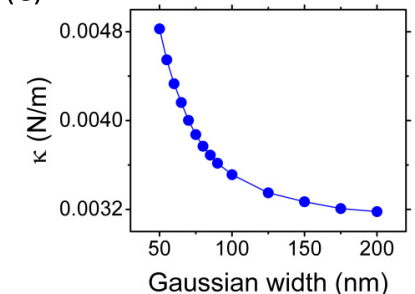

(e)

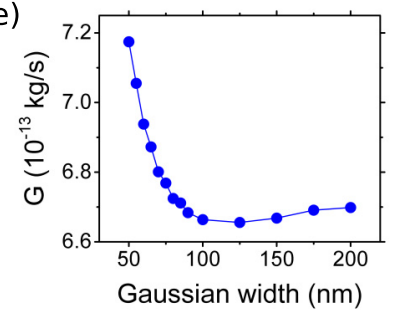

FIG. 2. (a) Simulated and calculated gyrotropic mode frequencies, $f_{G}$ [the latter calculated using Eq. (1)], in the presence of $200 \mathrm{mT}$ amplitude Gaussian [Eq. (2)] out-of-plane fields of various widths (the width is approximately 1.2 times the half-width at half-maximum (HWHM) of the Gaussian field profile). The simulated frequency for a spatially uniform $200 \mathrm{mT}$ out-of-plane field is shown by the gray dashed line. (b) Vortex core displacement as a function of static in-plane field for two Gaussian fields and zero out-of-plane field. (c) Stiffness coefficient versus Gaussian field width. (d) Gyroconstant calculated considering different radii from the disk center for three different Gaussian field widths. (e) Gyroconstant dependence on Gaussian field width where $G$ is calculated considering only the vortex structure within $40 \mathrm{~nm}$ of the disk's center.

The influence of $H_{\text {loc }}$ on the $H_{\mathrm{IP}}$-induced shift is visualized directly in Fig. 3 where we compare the equilibrium static positions of the vortex core (white) for $H_{\mathrm{IP}}=12 \mathrm{mT}$ in two cases: a vortex with no out-of-plane field [Fig. 3(a)] and a vortex subject to a $H_{\text {loc }}$ field with $w_{\text {loc }}=25 \mathrm{~nm}$ [Fig. 3(b)]. The core has clearly been displaced a smaller distance for the case of a localized field, with the core remaining within the strong part of the $H_{\text {loc }}$ profile (visible as a broad out-of-plane magnetization component at the disk's center). Reference images of the unshifted vortex core for both cases at $H_{\mathrm{IP}}=0 \mathrm{mT}$ are given in Figs. 3(c) and 3(d).

By looking at Fig. 3(d) and Fig. 4, the latter showing profiles of the out-of-plane component of the magnetization across the disk, one also sees that the presence of $H_{\mathrm{loc}}$ clearly modifies 


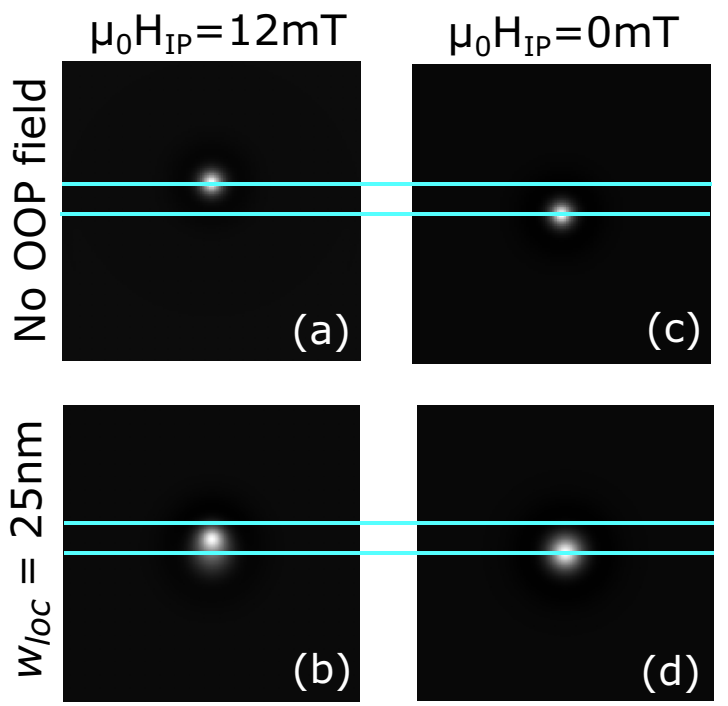

FIG. 3. Visualizations of the out-of-plane component of the disk magnetization (white) for (a) $\mu_{0} H_{\mathrm{uni}}=0 \mathrm{mT}$ and $\mu_{0} H_{\mathrm{IP}}=12 \mathrm{mT}$, (b) a $H_{\mathrm{loc}}$ with $w_{\mathrm{loc}}=25 \mathrm{~nm}$ and $\mu_{0} H_{\mathrm{IP}}=12 \mathrm{mT}$, (c) $\mu_{0} H_{\mathrm{uni}}=0 \mathrm{mT}$ and $\mu_{0} H_{\mathrm{IP}}=0 \mathrm{mT}$, and (d) a $H_{\mathrm{loc}}$ with $w_{\text {loc }}=25 \mathrm{~nm}$ and $\mu_{0} H_{\mathrm{IP}}=0 \mathrm{mT}$. The light blue lines reference the centered and $H_{\mathrm{IP}}$-displaced core positions in zero out-of-plane field. In (a) and (c) the white part of the image corresponds to the core. In (b) and (d) the out-of-plane core magnetization is convoluted with the $H_{\text {loc }}$-induced out-of-plane canting in the disk's center (which also translates to white coloring).

the magnetic configuration within the disk. For small $w_{\text {loc }}$ the equilibrium core structure itself is changed [Fig. 4(a)]: the magnetostatic halo is less sharp and the core widens. Intermediate $w_{\text {loc }}$ values [Figs. 4(b) and 4(c)] generate a clear outof-plane magnetized region around the core while large $w_{\text {loc }}$ values [Fig. 4(d)], which lead to a broad field profile, result in a magnetization profile which is similar to that seen for uniform out-of-plane fields [Fig. 1(e)]. For small displacements of the core in all of these cases the confining potential nevertheless (a)

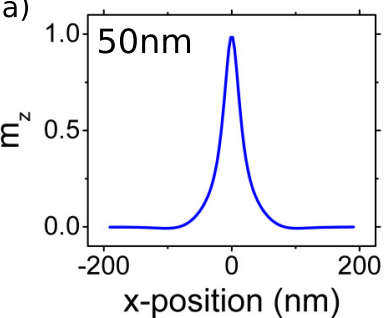

(c)

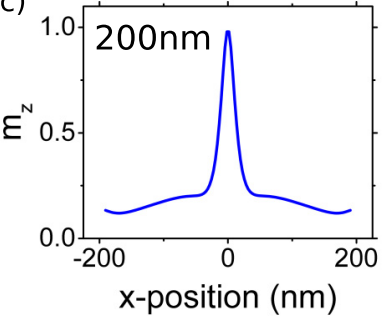

(b)

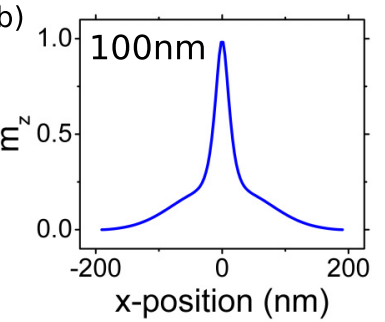

(d)

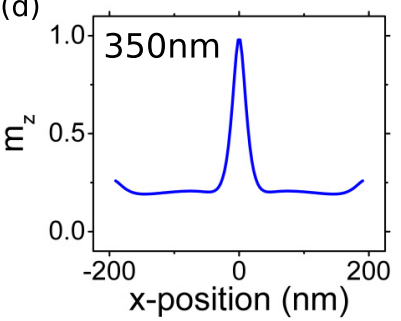

FIG. 4. Plots of the out-of-plane magnetization for a slice through the disk center for a vortex in Gaussian fields of width $w_{\text {loc }}$ of (a) $50 \mathrm{~nm}$, (b) $100 \mathrm{~nm}$, (c) $200 \mathrm{~nm}$, and (d) $350 \mathrm{~nm}$. remains close to harmonic and we have again used the method previously described to calculate (dynamic) values of $\kappa$ in the presence of localized fields. Consistent with the results seen in Figs. 2(a), 2(b), and 3, $\kappa$ increases strongly for narrower localizations of the Gaussian field [as shown in Fig. 2(c)], again confirming the $H_{\text {loc }}$-induced confinement.

We also calculated the gyroconstant in the presence of the $H_{\text {loc }}$ fields, again considering different radii as done previously. The resultant data are shown in Fig. 2(d) for three Gaussian field profiles. Notably, the peak in $G$ which was clearly visible in Fig. 1(c) disappears (or for $w_{\text {loc }}=200 \mathrm{~nm}$ becomes much less prominent) due to the absence of a deep magnetostatic halo for the studied $w_{\text {loc }}$ values (Fig. 4). For the two broader Gaussians, $G$ increases with the considered radius since the nonuniform $H_{\text {loc }}$ induces a canting which depends on the distance from the center of the disk [as seen in Figs. 4(b) and 4(c)]. For the narrowest Gaussian, $G$ becomes flat at large considered radii. This is because the narrow localization of $H_{\text {loc }}$ leads to a quasi-null-canting of $\mathbf{m}$ away from the center. However, $G$ grows quickly at small and intermediate considered radii, due to the $H_{\text {loc }}$-induced broadening of the core [Fig. 4(a)]. In Fig. 2(e) we show the extracted $G$ values versus $w_{\text {loc }}$ where $G$ was again calculated using a considered radius of $40 \mathrm{~nm}$ (i.e., analyzing the magnetization in the core's immediate vicinity). Like $\kappa, G$ also increases at small $w_{\text {loc }}$ but to a lesser extent.

The extracted $G$ and dynamic $\kappa$ yield reasonable quantitative agreement between the simulated frequencies and the frequency predicted by Eq. (1) [Fig. 2(a)]. This said, the agreement is clearly best at large $w_{\text {loc }}$. This is perhaps not unexpected however since wider profiles result in weaker deformation of the magnetization near the core. We also emphasize that, in contrast to the case of a uniform out-of-plane field the growth of $f_{G}$ at small $w_{\text {loc }}$ [Fig. 2(a)] is driven by increased confinement [i.e., $\kappa$ in Fig. 2(c)] rather than by changes in $G$ [Fig. 2(e)].

Up until now we have considered only the modifications to $f_{G}$ induced by changing $w_{\text {loc }}$. Figure 5 however shows data analogous to that in Fig. 1(a), demonstrating the change in $f_{G}$ induced when modifying the amplitude of the localized fields. The change to $f_{G}$ per unit of field amplitude, which can be thought of as a "field sensitivity," is notably more than five times larger for a localized Gaussian field with $w_{\text {loc }}=50 \mathrm{~nm}$ than that observed for a spatially uniform field. Consistent with the results shown in Fig. 2(a), this sensitivity enhancement reduces as the field profile is made broader (i.e., when $w_{\text {loc }}$ increases).

Finally for this section, we demonstrate that the trends observed in Fig. 2(a) apply to other disk geometries [57]. Figure 6(a) shows the frequency increase (relative to $f_{G}$ under $H_{\text {uni }}=200 \mathrm{mT}$ ) as a function of $w_{\text {loc }}$ for three different disk radii. The increase in $f_{G}$ is found to be almost independent of the disk radius. This demonstrates for these radii that the frequency increase (at least for small oscillations in the presence of this strong $200 \mathrm{mT}$ localized field) is largely independent of the "intrinsic" core confinement which is defined by the disk geometry. The increase instead results from the interaction between the localized field and the vortex core, the size of the latter being independent of the lateral disk dimensions [58]. Along these same lines, since $f_{G}$ is 


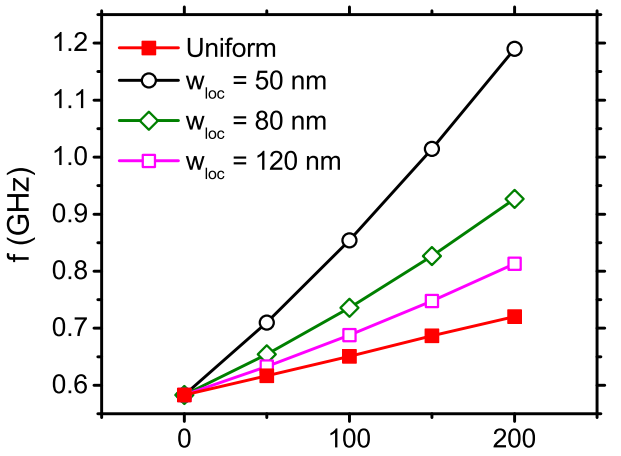

(Localized) out of plane field amplitude (mT)

FIG. 5. Simulated gyrotropic mode frequency plotted against the amplitude of localized, Gaussian fields with different widths. Data for a spatially uniform field [taken from Fig. 1(a)] are shown for comparison.

intrinsically smaller at larger disk radii $[15,18,19,45]$ (due to a weaker core confinement), the relative frequency shift induced by a localized field (i.e., as a percentage) will be higher for wider disks [see Fig. 6(b)].

Analogous behavior to that shown in Fig. 2(a) was also seen when reducing the disk thickness. The absolute change in $f_{G}$ did however reduce as the disk became thinner [Fig. 6(c)]. This reduction was found to be driven by a reduced dynamic $\kappa$ (confirmed at $w_{\text {loc }}=90 \mathrm{~nm}$ ), indicating a lower Gaussianfield-induced core confinement at smaller thicknesses. This reduced confinement is perhaps not surprising as decreasing the disk thickness leads to a smaller vortex core volume (the core is narrower [58] and its height reduces), lowering the $H_{\text {loc }}$-associated Zeeman energy which drives the confinement.

\section{B. Dipole fields and width-dependent Gaussian fields}

Localized magnetic fields can also be generated by uniformly magnetized, spherical MNPs. The in-plane components of the field generated by a submicron particle have previously been used to shift the vortex core position and probe the anharmonicity of the disk's intrinsic confining potential [56]. Here we consider the case of a field generated by a centralized MNP with radius $R_{\mathrm{P}}$ whose lower surface is at a fixed distance $(10 \mathrm{~nm})$ from the upper surface of the disk (i.e., the height of the center of the MNP from the top of the disk will be $R_{\mathrm{P}}+10 \mathrm{~nm}$ ). To minimize simulation time the field created by a MNP with saturation magnetization $M_{\mathrm{S}, \mathrm{P}}$ has been modeled as that of a dipole with moment $\frac{4}{3} \pi R_{\mathrm{P}}^{3} M_{\mathrm{S}, \mathrm{P}}$ where $M_{\mathrm{S}, \mathrm{P}}=200$ $\mathrm{kAm}^{-1}$. To confirm the validity of this simplification, we determined the gyrotropic frequency observed when a solid ferromagnetic sphere of diameter $100 \mathrm{~nm}\left(A_{\mathrm{ex}}=13 \mathrm{pJ} / \mathrm{m}\right)$ was placed above the center of the disk (in this case we used a smaller disk with $R=96 \mathrm{~nm}$ to minimize simulation time and memory use) and explicitly simulated it together with the disk in a $200 \mathrm{mT}$ uniform out-of-plane field. When compared to the simulated $f_{G}$ in an equivalent dipole field there was a relatively small discrepancy of $\approx 2.6 \%$ which was reduced to $0.3 \%$ when adding a strong $z$-axis-oriented anisotropy to the sphere $\left(K_{1}=10^{7} \mathrm{~J} / \mathrm{m}^{3}\right)$. The latter tends to fix the sphere's magnetization in the out-of-plane orientation, suggesting that the worse agreement for the zero-anisotropy sphere was due to changes in the sphere's magnetic configuration induced by the magnetic stray field of the disk. We also note that the simulations below were performed with no external out-ofplane field. However, a field would usually have to be applied experimentally in the case of a superparamagnetic particle and indeed we found analogous results to those given below [ $f_{G}$ versus $R_{\mathrm{P}}$ in Fig. 7(a)] for simulations in a $200 \mathrm{mT}$ external out-of-plane field. Such a field would be sufficient to induce the aforementioned $M_{\text {S,P }}$ value [30].

In Fig. 7(a), we show results obtained for dipoles equivalent to $+z$-magnetized MNPs of various sizes positioned above the disk as detailed above. In contrast to the case of the Gaussian field, $f_{G}$ displays a maximum at some intermediate particle size. This behavior cannot be explained simply by the stray field changing in magnitude as $R_{\mathrm{P}}$ changes. Indeed, if we run simulations to determine $f_{G}$ in the presence of uniform out-of-plane fields equivalent in magnitude to the MNP field (as calculated in the center of the disk) we see a monotonically increasing $f_{G}$ [red squares in Fig. 7(a)] with no peak. This growth in $f_{G}$ is consistent with the bigger particles generating stronger fields and thus larger changes in $f_{G}$ [i.e., as per Fig. 1(a)]. Instead, the peak in $f_{G}$ observed for the dipole fields at intermediate $R_{\mathrm{P}}$ can be correlated with a maximum in the confinement of the core, manifested as a peak in $\kappa$ [Fig. 7(b)]. Note however that the maximum $f_{G}$ occurs at a slightly higher diameter than that which leads to the maximum $\kappa$ due to the diameter dependence of $G$ which increases [thus (a)

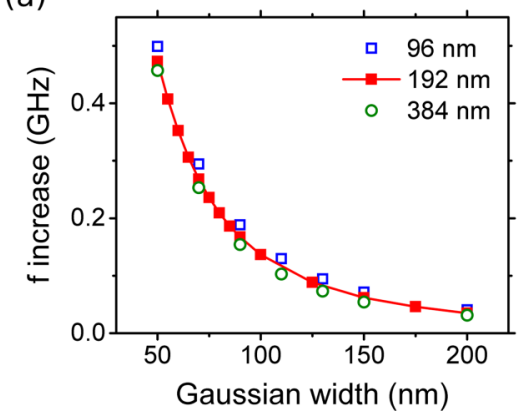

(b)

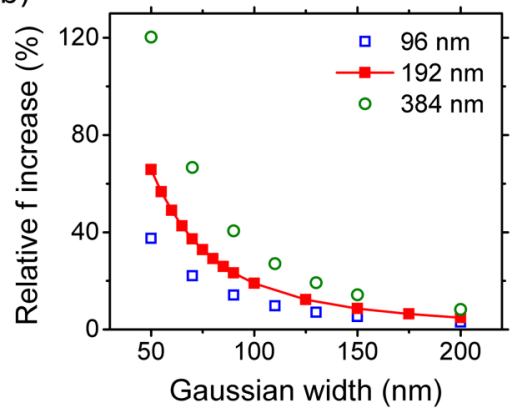

(c)

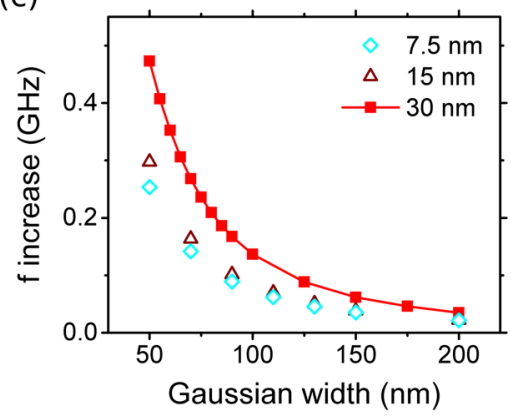

FIG. 6. The (a) absolute and (b) relative change in the gyrotropic frequency $f_{G}$ (compared to its value in $H_{\text {uni }}=200 \mathrm{mT}$ ) as a function of Gaussian width for disks of radii $R=96,192$, and $384 \mathrm{~nm}$ and a constant thickness of $L=30 \mathrm{~nm}$. (c) The absolute change in $f_{G}$ for disks of thickness $L=7.5,15$, and $30 \mathrm{~nm}$ and a constant radius of $R=192 \mathrm{~nm}$. 
(a)

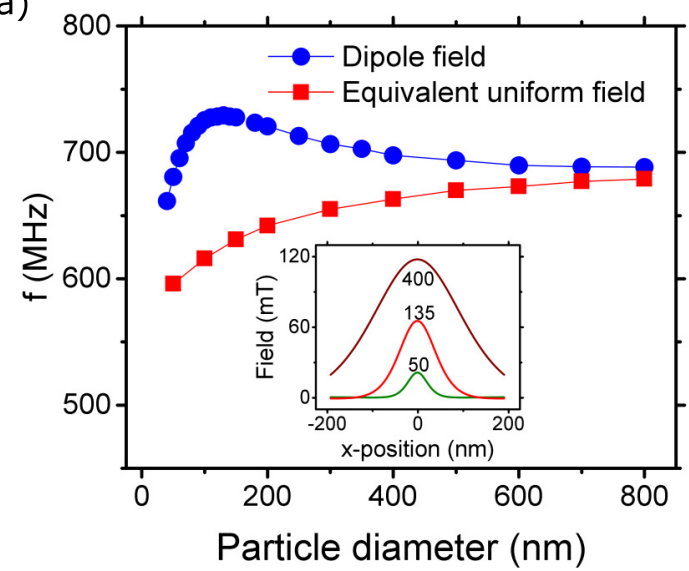

(b)

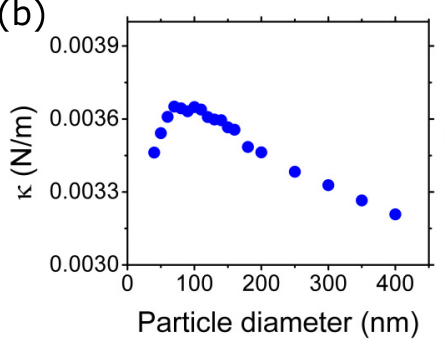

(c)

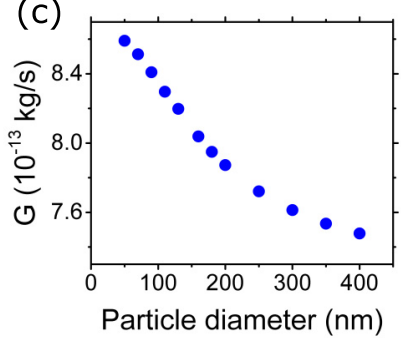

FIG. 7. (a) Simulated frequency as a function of MNP diameter $\left(2 R_{\mathrm{P}}\right)$. The red squares shows the simulated frequency in a uniform out-of-plane field of the same amplitude as the stray field created by the MNP as calculated at the disk center. The inset shows the out-ofplane component of the dipole field for three particle diameters below, above, and near the observed peak in $f_{G}$. (b) Stiffness coefficient as a function of particle diameter. (c) Gyroconstant calculated by considering the spin structure within $40 \mathrm{~nm}$ of the disk center as a function of particle diameter.

reducing $f_{G}$, as per Eq. (1)] as the particle diameter becomes small [Fig. 7(c)].

In the inset of Fig. 7(a) we show the out-of-plane component of the dipolar MNP field for particle diameters located around the point at which the peak in $f_{G}$ lies. The peak in confinement can be understood as follows. At small particle diameters (e.g., $2 R_{\mathrm{P}}=50 \mathrm{~nm}$ ), the field is highly localized; however the magnetic moment of the MNP (and thus the stray field amplitude) is low. This results in a weak confinement. A weak confinement also occurs for large particles (e.g., $2 R_{\mathrm{P}}=400 \mathrm{~nm}$ ) which generate strong but nevertheless broad (and thus weakly localized) spatial field profiles. Between these two extrema however is some intermediate particle size (e.g., $2 R_{\mathrm{P}}=135 \mathrm{~nm}$ ) where there is an optimal combination of field strength and localization which maximizes the core confinement, thus leading to a large $f_{G}$.

We can reproduce the above tendencies using Gaussian fields which have been scaled by a factor $\propto w_{\text {loc }}^{3} /\left(w_{\text {loc }}+d\right)^{3}$. This scaling mimics some characteristics of the $R_{\mathrm{P}}$-dependent dipole field. $d$ is chosen to be $25 \mathrm{~nm}$ as this is the distance used in our simulations between the bottom of the particle and center of the disk. The numerator in the above scaling factor describes the magnetic moment dependence on particle radius (here equivalent to $w_{\text {loc }}$ ) whereas the denominator describes the field behavior as the dipole center moves farther away

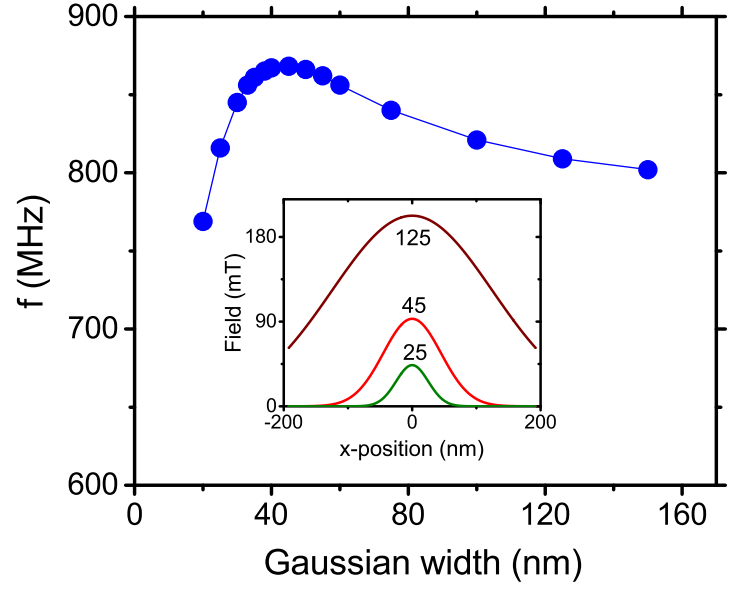

FIG. 8. Simulated frequency in the presence of a Gaussian field whose amplitude has been modified to depend on its width and thus generate analogous data to that in Fig. 7(a). Inset shows a central slice through the field profile for three different Gaussian widths.

from the disk center due to an increasing particle radius. The simulated $f_{G}$ values are shown in Fig. 8. Unlike the results for Gaussian fields of constant amplitude, $f_{G}$ now exhibits a peak at intermediate $w_{\text {loc }}$ values, analogous to the frequency behavior seen for MNPs when changing $R_{\mathrm{P}}$ [Fig. 7(a)]. Slices of the Gaussian field profiles are shown in the inset of Fig. 8 for three different $w_{\text {loc }}$ values. Again, as we increase $w_{\text {loc }}$ we see a transition from a weak, highly localized field to a strong, broadly localized field with maximum confinement occurring at an intermediate $w_{\text {loc }}$.

\section{CONCLUSION}

We have shown that the sensitivity of the vortex gyrotropic mode frequency to out-of-plane fields can depend strongly on the spatial localization of those fields. Centralized, outof-plane magnetic fields localized over lengths which are comparable to or a few times larger than the vortex core radius induce significantly larger changes in the vortex gyrotropic frequency than that generated by a spatially uniform out-ofplane field of the same amplitude. This behavior is consistent with an increase in the vortex stiffness as a result of the out-of-plane magnetization of the core preferentially aligning with the strongest part of the localized field which generates an additional vortex core confinement. In the case of fields which approximate those generated by magnetic particles of varying radius, the frequency was observed to be maximized for some intermediate particle size which led to an optimized combination of field amplitude and field localization. This may be relevant for vortex-based MNP sensors exploiting changes of the gyrotropic frequency induced by localized MNP fields [59]. We also emphasize that this work has focused on very low amplitude excitations and highlight the fact that gyrotropic motion outside of the strongest part of the localized field will result in weaker changes to $f_{G}$ (as observed by Min et al. [40] for large-amplitude oscillations around pinning sites generated by changes in saturation magnetization). Finally we note that the "disk-on-a-disk" structure recently studied by Ding et al. [33] may offer a way to generate centralized, localized 
out-of-plane fields; however this would require the upper disk to be made from a material with out-of-plane magnetic anisotropy, ideally with a nonmagnetic spacer between the two disks in order to minimize interlayer exchange coupling (e.g., as per [60]).

\section{ACKNOWLEDGMENTS}

This work was supported by the Australian Research Council's Discovery Early Career Researcher Award scheme (DE120100155), a research grant from the United States Air
Force Asian Office of Aerospace Research and Development (AOARD), the University of Western Australia (including the ECRFS, RCA, and RDA schemes), NeCTAR (National eResearch Collaboration Tools and Resources, supported by the Australian Government through the National Collaborative Research Infrastructure Strategy), and resources provided by the Pawsey Supercomputing Centre with funding from the Australian Government and the Government of Western Australia. The authors thank Joo-Von Kim, Manu Sushruth, Mikhail Kostylev, Vincent Cros, Rebecca Carey, Maximilian Albert, and Hans Fangohr for useful discussions, advice, and/or assistance.
[1] R. P. Cowburn, D. K. Koltsov, A. O. Adeyeye, M. E. Welland, and D. M. Tricker, Phys. Rev. Lett. 83, 1042 (1999).

[2] T. Shinjo, T. Okuno, R. Hassdorf, K. Shigeto, and T. Ono, Science 289, 930 (2000).

[3] A. Wachowiak, J. Wiebe, M. Bode, O. Pietzsch, M. Morgenstern, and R. Wiesendanger, Science 298, 577 (2002).

[4] K. Y. Guslienko and V. Novosad, J. Appl. Phys. 96, 4451 (2004).

[5] A. Vogel, T. Kamionka, M. Martens, A. Drews, K. W. Chou, T. Tyliszczak, H. Stoll, B. Van Waeyenberge, and G. Meier, Phys. Rev. Lett. 106, 137201 (2011).

[6] S. Yakata, T. Tanaka, K. Kiseki, K. Matsuyama, and T. Kimura, Sci. Rep. 3, 3567 (2013).

[7] V. S. Pribiag, I. N. Krivorotov, G. D. Fuchs, P. M. Braganca, O. Ozatay, J. C. Sankey, D. C. Ralph, and R. A. Buhrman, Nat. Phys. 3, 498 (2007).

[8] A. Dussaux, B. Georges, J. Grollier, V. Cros, A. V. Khvalkovskiy, A. Fukushima, M. Konoto, H. Kubota, K. Yakushiji, S. Yuasa, K. A. Zvezdin, K. Ando, and A. Fert, Nat. Commun. 1, 8 (2010).

[9] A. S. Jenkins, R. Lebrun, E. Grimaldi, S. Tsunegi, P. Bortolotti, H. Kubota, K. Yakushiji, A. Fukushima, G. de Loubens, O. Klein, S. Yuasa, and V. Cros, Nat. Nanotechnol. (2016), doi: 10.1038/nnano.2015.295.

[10] D. Kim, E. Rozhkova, I. Ulasov, S. Bader, T. Rajh, M. Lesniak, and V. Novosad, Nat. Mater. 9, 165 (2010).

[11] K. Yamada, S. Kasai, Y. Nakatani, K. Kobayashi, H. Kohno, A. Thiaville, and T. Ono, Nat. Mater. 6, 270 (2007).

[12] R. Huber and D. Grundler, Proc. SPIE 8100, 81000D (2011).

[13] D.-S. Han, A. Vogel, H. Jung, K.-S. Lee, M. Weigand, H. Stoll, G. Schütz, P. Fischer, G. Meier, and S.-K. Kim, Sci. Rep. 3, 2262 (2013).

[14] C. Behncke, M. Hänze, C. F. Adolff, M. Weigand, and G. Meier, Phys. Rev. B 91, 224417 (2015).

[15] K. Y. Guslienko, B. A. Ivanov, V. Novosad, Y. Otani, H. Shima, and K. Fukamichi, J. Appl. Phys. 91, 8037 (2002).

[16] S.-B. Choe, Y. Acremann, A. Scholl, A. Bauer, A. Doran, J. Stöhr, and H. A. Padmore, Science 304, 420 (2004).

[17] B. A. Ivanov, J. Appl. Phys. 95, 7444 (2004).

[18] K. Y. Guslienko, X. F. Han, D. J. Keavney, R. Divan, and S. D. Bader, Phys. Rev. Lett. 96, 067205 (2006).

[19] G. de Loubens, A. Riegler, B. Pigeau, F. Lochner, F. Boust, K. Y. Guslienko, H. Hurdequint, L. W. Molenkamp, G. Schmidt,
A. N. Slavin, V. S. Tiberkevich, N. Vukadinovic, and O. Klein, Phys. Rev. Lett. 102, 177602 (2009).

[20] N. Locatelli, V. V. Naletov, J. Grollier, G. de Loubens, V. Cros, C. Deranlot, C. Ulysse, G. Faini, O. Klein, and A. Fert, Appl. Phys. Lett. 98, 062501 (2011).

[21] K. S. Buchanan, P. E. Roy, M. Grimsditch, F. Y. Fradin, K. Y. Guslienko, S. D. Bader, and V. Novosad, Phys. Rev. B 74, 064404 (2006).

[22] J.-V. Kim, Solid State Phys. 63, 217 (2012).

[23] R. Lebrun, N. Locatelli, S. Tsunegi, J. Grollier, V. Cros, F. Abreu Araujo, H. Kubota, K. Yakushiji, A. Fukushima, and S. Yuasa, Phys. Rev. App. 2, 061001 (2014).

[24] P. M. Braganca, B. A. Gurney, B. A. Wilson, J. A. Katine, S. Maat, and J. R. Childress, Nanotechnol. 21, 235202 (2010).

[25] R. J. Ryan, H. Xi, and I. Jin, US Patent No. 8053244 B2 (2011).

[26] T. Srimani, A. N. Mukhopadhyay, K. Roy, and M. Sharad, arXiv:1511.09072.

[27] K. Mizushima, K. Kudo, T. Nagasawa, and R. Sato, J. Appl. Phys. 107, 063904 (2010).

[28] M. Inoue, A. Baryshev, H. Takagi, P. B. Lim, K. Hatafuku, J. Noda, and K. Togo, Appl. Phys. Lett. 98, 132511 (2011).

[29] S. Atalay, A. O. Kaya, V. S. Kolat, H. Gencer, and T. Izgi, J. Supercond. Nov. Magn. 28, 2071 (2015).

[30] P. J. Metaxas, M. Sushruth, R. Begley, J. Ding, R. C. Woodward, I. Maksymov, M. Albert, W. Wang, H. Fangohr, A. Adeyeye, and M. Kostylev, Appl. Phys. Lett. 106, 232406 (2015).

[31] R. S. Gaster, D. A. Hall, C. H. Nielsen, S. J. Osterfeld, H. Yu, K. E. Mach, R. J. Wilson, B. Murmann, J. C. Liao, S. S. Gambhir et al., Nat. Med. 15, 1327 (2009).

[32] G. Heldt, M. T. Bryan, G. Hrkac, S. E. Stevenson, R. V. Chopdekar, J. Raabe, T. Thomson, and L. J. Heyderman, Appl. Phys. Lett. 104, 182401 (2014).

[33] J. Ding, P. Lapa, S. Jain, T. Khaire, S. Lendinez, W. Zhang, M. B. Jungfleisch, C. M. Posada, V. G. Yefremenko, J. E. Pearson, A. Hoffmann, and V. Novosad, arXiv:1601.03133.

[34] T. Uhlig, M. Rahm, C. Dietrich, R. Höllinger, M. Heumann, D. Weiss, and J. Zweck, Phys. Rev. Lett. 95, 237205 (2005).

[35] R. Zarzuela, S. Vélez, J. M. Hernandez, J. Tejada, and V. Novosad, Phys. Rev. B 85, 180401(R) (2012).

[36] R. Zarzuela, E. M. Chudnovsky, J. M. Hernandez, and J. Tejada, Phys. Rev. B 87, 144420 (2013). 
[37] R. L. Silva, A. R. Pereira, R. C. Silva, W. A. Moura-Melo, N. M. Oliveira-Neto, S. A. Leonel, and P. Z. Coura, Phys. Rev. B 78, 054423 (2008).

[38] A. Vansteenkiste, M. Weigand, M. Curcic, H. Stoll, G. Schütz, and B. Van Waeyenberge, New J. Phys. 11, 063006 (2009).

[39] R. L. Compton, T. Y. Chen, and P. A. Crowell, Phys. Rev. B 81, 144412 (2010).

[40] H. Min, R. D. McMichael, J. Miltat, and M. D. Stiles, Phys. Rev. B 83, 064411 (2011).

[41] T. Y. Chen, M. J. Erickson, P. A. Crowell, and C. Leighton, Phys. Rev. Lett. 109, 097202 (2012).

[42] A. Vansteenkiste, J. Leliaert, M. Dvornik, M. Helsen, F. Garcia-Sanchez, and B. Van Waeyenberg, AIP Adv. 4, 107133 (2014).

[43] B. A. Ivanov and C. E. Zaspel, Appl. Phys. Lett. 81, 1261 (2002).

[44] B. A. Ivanov and C. Zaspel, Phys. Rev. Lett. 94, 027205 (2005).

[45] J. P. Park and P. A. Crowell, Phys. Rev. Lett. 95, 167201 (2005).

[46] C. E. Zaspel, B. A. Ivanov, J. P. Park, and P. A. Crowell, Phys. Rev. B 72, 024427 (2005).

[47] F. G. Aliev, J. F. Sierra, A. A. Awad, G. N. Kakazei, D.-S. Han, S.-K. Kim, V. Metlushko, B. Ilic, and K. Y. Guslienko, Phys. Rev. B 79, 174433 (2009).

[48] M. J. Donahue and D. G. Porter, OOMMF User's Guide, Version 1.0, Interagency Report NISTIR 6376, Tech. Rep. (National Institute of Standards and Technology, Gaithersburg, MD, 1999).

[49] T. Fischbacher, M. Franchin, G. Bordignon, and H. Fangohr, IEEE Trans. Magn. 43, 2896 (2007).

[50] Simulations for an identically sized disk $(R=192 \mathrm{~nm}, t=$ $30 \mathrm{~nm}$ ) with a $3 \times 3 \times 3 \mathrm{~nm}^{3}$ cell size were run in OOMMF for zero field (agreement with MuMax3 within 1.1\%) and in the presence of a localized $H_{\text {loc }}$ field (as in Sec. IV A) with $w_{\text {loc }}=100 \mathrm{~nm}$ (agreement within 1\%). There was also good agreement in the bare disk frequency using the eigenmode solver in FinMag for a $R=96 \mathrm{~nm}$ disk; however a larger meshing had to be used at the disk edges due to memory constraints.

[51] A. Thiele, Phys. Rev. Lett. 30, 230 (1973).

[52] D. L. Huber, Phys. Rev. B 26, 3758 (1982).

[53] B. A. Ivanov and C. E. Zaspel, Phys. Rev. Lett. 99, 247208 (2007).

[54] We observed a $0.4 \%$ change in $G$ across different layers of the disk.

[55] Y. Gaididei, V. P. Kravchuk, and D. D. Sehka, Int. J. Quantum Chem. 110, 83 (2010).

[56] O. V. Sukhostavets, B. Pigeau, S. Sangiao, G. de Loubens, V. V. Naletov, O. Klein, K. Mitsuzuka, S. Andrieu, F. Montaigne, and K. Y. Guslienko, Phys. Rev. Lett. 111, 247601 (2013).

[57] Other disk geometries were simulated using a cell size of $3 \times 3 \times 3.75 \mathrm{~nm}^{3}$.

[58] N. Usov and S. Peschany, J. Magn. Magn. Mater. 118, L290 (1993).

[59] P. Wohlhüter, M. T. Bryan, P. Warnicke, S. Gliga, S. E. Stevenson, G. Heldt, L. Saharan, A. K. Suszka, C. Moutafis, R. V. Chopdekar, J. Raabe, T. Thomson, G. Hrkac, and L. J. Heyderman, Nat. Commun. 6, 7836 (2015).

[60] P. J. Metaxas, P. J. Zermatten, R. L. Novak, S. Rohart, J. P. Jamet, R. Weil, J. Ferré, A. Mougin, R. L. Stamps, G. Gaudin, V. Baltz, and B. Rodmacq, J. Appl. Phys 113, 073906 (2013). 